Military Technical College Kobry El-Kobba

Cairo, Egypt

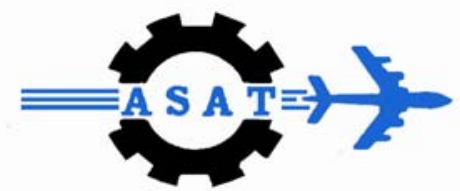

12-th International Conference

on

Aerospace Sciences \&

Aviation Technology

\title{
A CDMA ROUTE PROTOCOL FOR AD HOC WIRELESS NETWORKS
}

\author{
FAYEZ W. ZAKI* and NAGY Y. HASSAN**
}

\begin{abstract}
A Mobile ad hoc network is a collection of wireless nodes, all of which may be mobile, laptop , personal digital assistants, that dynamically create a network without using any infrastructure. Each host participating in the network acts both as a host and as a router. As mobile hosts are no longer just end systems; each node must be able to function as a router as well as to relay packets generated by other nodes. As the nodes move in and out with respect to each other, the resulting changed topology must somehow be able to communicate to all other nodes as appropriate. In such networks, it is of a special importance to grantee Quality of Service (QoS), such as packet loss and bandwidth. To accomplish this, an admission control scheme which guarantee bandwidth for real-time applications in multihop mobile ad hoc networks is developed. In this scheme there is end-to-end bandwidth calculation and bandwidth allocation. To perform this objective, the source is informed of the bandwidth and QoS available to any destination in the network to enable the establishment of QoS connections and the efficient support of real time applications in the network.

Simulation results showed distinct performance advantages of the introduced protocol over those reported previously.
\end{abstract}

\section{KEY WORDS}

Ad hoc wireless networks, code division multiple access(CDMA), quality-of-service (QoS)

${ }^{*}$ Professor , Dept. of Electronics and Communication Eng. , Faculty of Eng. Mansoura University, Egypt.

${ }^{* *}$ Graduate student, Dept. of Electronics and Communication Eng. , Faculty of Eng. Mansoura University, Egypt. 


\section{INTRODUCTION}

Routing protocols for traditional wired networks are designed to support tremendous numbers of nodes, but they assume that the relative position of the nodes are generally remain unchanged. On the contrary, in mobile networks there may be fewer which to route, however the network topology changes may be drastic and frequent as the mobile nodes move.

Efficient routing in multihop mobile network requires that the routing protocol operate in an on-demand fashion. Moreover, the routing protocol must limit the number of nodes that should be informed of topology changes. The challenge in the design of this mobile network is to develop a dynamic routing protocols that can find routes between two communicating mobile hosts (nodes) and should be able to keep up with the high degree of node mobility.

Communication between hosts in the network can be multihop wireless links. The ad hoc mobile host also acts as a router, it forwards data packets to other hosts. The implementation of this network can be wireless local area network or the cellular networks (e.g., GPRS, UMTS or IMT-2000). Such network usually connected to wired network, e.g., ATM or Internet, so that the mobile users can be connected to Internet multimedia. There were different forms which have appeared in the wireless extensions of the wired ATM[1]. The difficulty here is to guarantee QoS parameters ( in wireless part ) through all the path when connecting a multihop wireless network to wired backbone network.

In this paper the bandwidth is considered as a measure of QoS. In time-slotted network systems the bandwidth is measured by the number of time slots. The aim of QoS routing is to find the shortest available path that achieves the required bandwidth. The protocol reported in [2] finds the shortest path and then check the required bandwidth. If it happens that the bandwidth is unavailable the call will be rejected. On the other hand, the protocol reported in [3] finds the shortest path that provides the required bandwidth, i.e., the selected path may not be the shortest one . Moreover, it computes the bandwidth, determines and schedules the slots that will be used before the completion of the reservation process.

The protocol introduced here provides two advantages over that reported in [3]. That is the system also computes the route that support the required bandwidth and performs slot assignment during the reservation process. TDMA is used for bandwidth reservation in [3]. Here, to improve the system performance CDMA is overlaid upon TDMA and used for bandwidth reservation, i.e., more than one session can share the same slot.

\section{QOS DEFINATION AND BANDWIDTH CALCULATION}

In cellular single hop system, it may be easier to guarantee the QoS parameters than in multihop system. The QoS parameters should be propagated through the network if the ATM VC (virtual circuit) will be extended to the wireless network. The resources must be reserved and controlled to guarantee the QoS through the 
network. The difficulty in multihop multimedia networks is to account the resources so that bandwidth reservations can be performed efficiently. For QoS for real-time applications support, the minimal hop-distance path to the destination that support the available bandwidth must be known.

Here consideration is only on "bandwidth" as the QoS parameter (ignoring Signal to Interference Ratio (SIR), packet loss rate, etc. It is due the importance of bandwidth guarantee for real time applications. In TDMA (time-slotted) network systems the "Bandwidth" can be translated into the number of free slots. The objective of the QoS routing is to select the shortest path among all paths which satisfy the bandwidth condition.

A call admission control based on on-demand routing protocol for QoS support in multihop mobile networks is proposed. In this protocol, no routing information and no routing tables are exchanged periodically. For performance evaluation different QoS traffic flows in the network is considered.

In the protocol reported here, it is concentrated on finding a feasible bandwidth route that satisfy the bandwidth demand whether it satisfies the shortest path or not. However, the protocol finds the shortest path of the available feasible routes. Moreover no routing tables are used in the protocol introduced here.

In this protocol, the transmission time scale is organized in frames. Each frame consists of fixed number of time slots as shown in Fig.1. The entire network is synchronized on a frame and slot basis. The frame/slot synchronization mechanism is not described here, but can be implemented in the mobile ad hoc networks with techniques similar to those employed in the wired networks [4]. Slot synchronization may be imprecise because of propagation delays in wireless ad hoc mobile environment. To overcome this problem slot guard times (fractions of a microsecond) are taken into consideration to kill the effect of propagation delay. Referring back to Fig.1, it can be seen that each frame is divided into control phase and data phase, all the control functions such as slot and frame synchronization, power measurement, code assignment, VC setup, slots request are performed in the control phase. The control phase uses pure TDMA. Each node in its turn broadcasts its information to all of its neighbors that can hear, such that the network control functions can be performed distributively. The information transmitted are assumed to be heard by adjacent nodes. An acknowledgment scheme is carried out to be suitable for noisy environment, in which the information may not be transmitted correctly to the neighbors. In control slot, each node has to ACK for the last information. Exploiting this approach, after issuing the data slot reservation, there may be one frame delay for the data transmission.

At the control phase end, the channel reservation status of the data phase will be transmitted to all nodes. This information will help to know free slots. 


\subsection{CDMA over TDMA and Hidden Terminal Problem}

Consider the wireless network shown in Fig.2 which uses TDMA for data transmission. A mobile host $A$ requires to transmit data to mobile host $D$. Assume all the slots in the TDMA frame are free and slots (1\&2) are reserved to transmit data to mobile host $B$ and slots ( $3 \& 4)$ to forward packets to the next node in the route (mobile host $C$ ). The mobile host $C$ may use slots $(1 \& 2)$ to forward packets to destination (mobile host $\mathrm{D}$ ). Here a collision at $\mathrm{B}$ may occur (because $\mathrm{B}$ may receive packets from $A$ and $C$ in the same time) [6]. The solution to solve the problem can be achieved by using CDMA (all spreading codes are orthogonal to each other). Different codes can be assigned to each node to transmit in the same time on slots used before without any collision. That is, each transmitter- is assigned a code for data transmission. A spreading code can be reused if two nodes have a hop distance greater than two [6]. In Fig.3, it can be seen that the same slots (1 and 2) are used to send packets encoded by a different code without any collision at $B$.

In simulation study, a TDMA is assumed first then CDMA is overlaid upon TDMA is considered and comparison is carried out. As shown in Fig.3, ideal code assignment scheme [2] is assumed running in the lower layer of the system, and all spreading codes are completely orthogonal to each other. Thus, the hidden terminal problem is avoided.

Link bandwidth is the set of the common free slots between two adjacent nodes as the network is multihop and only adjacent nodes can hear the reservation information. To compute the bandwidth from $C$ to $A$ as in Fig.4, consider the next hop is $B$. If $B$ can compute the bandwidth to $A$, then $C$ can use this information and the "link bandwidth" to $B$ to compute the path bandwidth to $A$. The link bandwidth may be estimated as follow:

link_BW $(P, Q)=$ free_slot $(P) \cap$ free_slot( $Q)$.

Path bandwidth (the end-to-end bandwidth) between two nodes is the set of available slots between them. The two nodes may be adjacent or not. If they are adjacent, the path bandwidth is the link bandwidth. If $C$ has free slots $\{2,3,5,6\}$, and $B$ has free slots $\{0,2,3,6\}$, then the link bandwidth between $C$ and $B$ is $\{2,3,6\}$. This means that only exploit slot 2 , slot 3 and slot 6 can be used for transmit packets from $C$ to $B$. Thus, if a VC session needs more than three slots, the call will be rejected.

\subsection{The On-Demand Routing with Bandwidth Constraint}

\subsubsection{Route discovery}

As in protocols based on on-demand rule like Ad-hoc On-demand Distance Vector routing (AODV) [1] and Dynamic Source Routing (DSR) [7], for communication between two hosts a route request (RREQ) packet must be broadcasts through the network. If there is a route between the source and the destination, a route request (RREQ) will record it. In the protocol reported here, all packets contain the following fields:

<packet_type, source_addr, dest_addr, sequence\#,route_list, data, TTL> 
The packet definition of different packet types in the above filed are shown in Table1. <source_addr, sequence\#> uniquely identifies a packet. This sequence\# is incremented when ever the source issues a new RREQ. Routing information are recorded in the route_list. When any node receives a RREQ, it will send a route reply (RREP) back to the source if satisfies the RREQ and computes the bandwidth from the source to this node. Otherwise it will rebroadcasts the RREQ to it's the neighbors after decrementing TLL by one. The node appends its address to the route_list. If TTL counts down to zero, the RREQ will be dropped and not to be processed it, TTL can limit the length of the number of hops for the path. There may exist a very long path which satisfies the bandwidth requirement. However, this path will be difficult to maintain within a dynamic environment. The use of TTL can control the flooding traffic. Note a node can receive more than one copy of the same route request broadcasted from various neighbors. If the address exists in the route list in the RREQ, this RREQ will be dropped (do not rebroadcast) and do not process it. Up to now data slots status is the same, no modification are made.

The destination node may receive more than one RREQ. Every RREQ represents a feasible QoS path from the source to the destination. Then the destination can choose the more suitable path and keep another paths to use if the first one fails. To reduce the overhead of broadcasting, the destination node can broadcast a CLEAN_RREQ packet to clean RREQ packets that are still roaming in the network after keeping required number of paths to avoid Multiple connectivity between a source - destination pair.

\subsubsection{Route reservation}

When the source node sends one RREQ packet to the destination node, the destination sends a route reply (RREP) travels backward to the source according to the route recorded in the route_list. It automatically sets up the reverse path from the destination back to the source. The destination can set up a bandwidth route and reserves slots hop-by-hop backward to the source according to the information recorded within the RREQ. The fields <route_list, > is copied from RREQ to RREP. As the RREP traverses back to the source, each node along the path calculates and reserves slots. When the source receives a RREP, the end-to-end bandwidth reservation is successful, and the virtual circuit (VC) is established. Then, the source node can begin transmitting datagrams. It is notable that the establishment of a VC between two nodes is a two-way handshaking. The call admission control begins this establishment protocol after a new call request. The call will be accepted if only the reservation process is successfully completed

\subsubsection{Unsuccessful reservation}

When the destination sends the RREP backward to the source, the reservation process may not succeed. The reason may be that the slots which the system wants to reserve are occupied a little earlier by another VC or the route break and there are no slots that can be replaced. If it happens, the rout must be stopped and the interrupted node sends a NACK (i.e., RESERVE_FAIL) to the destination to restart reservation process using another path that was kept before. All nodes on the route from the interrupted node to the destination must free the reserved data slots when receiving RESERVE_FAIL. If there is no VC that can be set along all feasible bandwidth routes, the destination broadcasts another NACK (i.e., NO_ROUTE) to 
notify the source. In case of receiving NO_ROUTE, the source can either re-start the discovery process, or reject the new call. In addition to NO_ROUTE arrival, if there is no any response back to the source before the timeout occurs, the source can also re-perform the route discover operation.

Once a VC is established, the source begin sending datagrams in the data phase. At the end of the session, all reserved slots must be released. These free slots will be contended by all new connections. However, if the last packet is lost, the reserved slots that are released will not known. This issue will be discussed in the next subsection.

\subsubsection{Connection breakage}

As a result of permanent change of the topology (due to node movement ) a VC may be destroyed. The system must rebuild another VC over a new path. When breakage happens send a special NACK (i.e., ROUTE_BROKEN) to the source and the destination, once the next hop becomes unreachable with two breakpoints, one of them sends a NACK to the source, and the other breakpoint sends a NACK to the destination. Each node along the path forwards this ROUTE_BROKEN to its neighbors and so on. Furthermore, they release all reserved slots for this connection and drop all data packets of this connection which are still waiting in the queue for transmission. Once the source receives the ROUTE_BROKEN, it re-starts the discovery process to re-establishs a VC over a new path, and the destination only drops the ROUTE_BROKEN (the main purpose of the travel of the ROUTE_BROKEN from the breakpoint to the destination is to release the reserved slots). The process can be repeated if there is any result until the completion of the call or timeout occurred which leads to data delivery for this session. The last data packet may be still on the way to the destination if a link on the VC is broken before the completion of session, can not reach the destination and is suspended within an intermediate node. The timeout scheme is used for each reserved slot. If a reserved slot is not used to deliver data packets for a couple of data frames and timeout occurs, this slot is set free automatically. Such free slots will be fully utilized by the other new sessions.

\section{PERRFORMANCE EVALUATION}

The simulated environment consists of 20 node and moving uniformly in $1000 \times 1000$ $\mathrm{ft}^{2}$ area. The nodes move at random directions, but uniform speed ( the simulation program supports three types of movement -random model, deterministic model and semi-deterministic model). The direction of each node is random and changes every $1 \mathrm{sec}$. Radio transmission range is $400 \mathrm{ft}$. That is, two nodes can hear each other if their distance is within the transmission range. Data rate is $2 \mathrm{Mbit} / \mathrm{s}$. In the simulation experiments, the channel quality may affect the packet transmission. That is, the noise in the channel may cause errors in packets. The channel quality specified by the bit error rate is uniform in all experiments. Because the VC traffic is delay sensitive rather than error sensitive, packets therefore are not ACKed. A coding scheme is assumed running in the system to do the forward error correction. 
In the experiments, more attention was paid to the effect of mobility upon the system performance. In each time frame (Fig.1), the data slot in the data phase is $5 \mathrm{~ms}$, and the control slot in control phase is $0.1 \mathrm{~ms}$. Channel overhead (e.g., code acquisition time, preamble, etc.) is factored into control/data packet length. It is assumed that there are 16 data slots in the data phase. So the frame length is $20 * 0.1+16 * 5=82$ ms. The source-destination pair of a call is randomly chosen and their distance must be greater than one. The randomizer can not permit to a call to be generated if this call existed in the last ten generated calls. When a call request is generated for a path, a transmission window(i.e. data slots) is reserved (on that link) automatically for all the subsequent packets in the connection. . The window is released when either the session is finished or the NACK packet (RESERVE_FAIL and ROUTE_BROKEN) is received. For a connection (call) the destination keeps three different bandwidth routes which are piggybacked within the first three RREQ's arriving at the destination. Simulation is carried out for three types of QoS for the offered traffic. QoS1, QoS2, and QoS4 which require one, two, and four data slots in each frame, respectively. For each simulation result, considering 10 different topologies and run 10000 frame time (i.e., 10000 * $82 \mathrm{~ms}$ ) for each topology. The interarrival time of two calls is an exponential distribution with the mean value 10 cycles $(820 \mathrm{~ms})$. Each session length of QoS1, QoS2, and QoS4 is 100, 200, and 400 packets, respectively. Constant bit rate is assumed in all traffics. The interarrival time of packets within QoS1 is one cycle $(82 \mathrm{~ms})$. Similarly, the interarrival times for QoS2 and QoS4 are $41 \mathrm{~ms}$ and $21 \mathrm{~ms}$, respectively.

In the first experiment, the effect of variable mobility on incomplete connection ratio is considered. A call may not be completed due to the mobility. If the last packet can arrive at the destination, this session is defined to be complete. Otherwise, it is incomplete. The calls are classified into complete, incomplete, rejected (that cant not find any routs or the three routs failed to be reserved) and incomplete at end ( this case occurs when the source finishes sending all the packets and the route is broken and the last packet can not arrive to the destination ). Some data packets of a complete session may be lost because of the topological change. The VC of a connection may be re-established during the active period and the last packet finally can get to the destination. This is also considered as a complete session. In the simulation, only the source is allowed to re-perform the route discovery operation once. If the session still can not be completed, it will be incomplete. Fig.5, Fig.6 and Fig.7 illustrate the result.

Observe that high mobility causes a path to be broken frequently. When mobility is $20 \mathrm{ft} / \mathrm{s}$ for example, QoS4, QoS2 and QoS1 respectively have 6.47\% , 5.57\% and $3.58 \%$ sessions which can not be completed. Using CDMA the results were improved to $2.98 \%, 3.78 \%, 2.35 \%$ respectively (that is the incomplete ratio decreases with $54 \%, 32 \%$ and $34 \%$ respectively). The bandwidth route of lower QoS traffic-as is to be expected, can be re-established easier.

In Fig.8, Fig.9 and Fig.10 it is noticed that the percentage of calls that were broken and be re-established is proportional to the mobility as expected.

Note that in case of QoS4 the rerouting percentage using CDMA is greater than pure TDMA, this is due to the performance enhancement that makes some calls that were broken find another routs. 


\section{CONCLUSIONS}

In summary, an admission control was presented over an on-demand routing protocol which is suitable for use with multihop mobile networks. This protocol is more powerful in the resource management than the work in [2,3]. Thus it can accept more calls in the network according to the simulation results. The route may not be the shortest in hop length. In the route reply process, performing slot assignment and the route reservation are made hop-by-hop backward from the destination to the source. This admission control can be applied to two important scenarios: multimedia ad-hoc wireless networks and multihop extension wireless ATM networks. In the performance experiments, traffic flows with different QoS types are considered. Finally, The protocol introduced here provides two advantages over that reported in [3]. That is the system also computes the route that support the required bandwidth and performs slot assignment during the reservation process. TDMA is used for bandwidth reservation and CDMA is also overlaid upon TDMA to improve the system performance. 


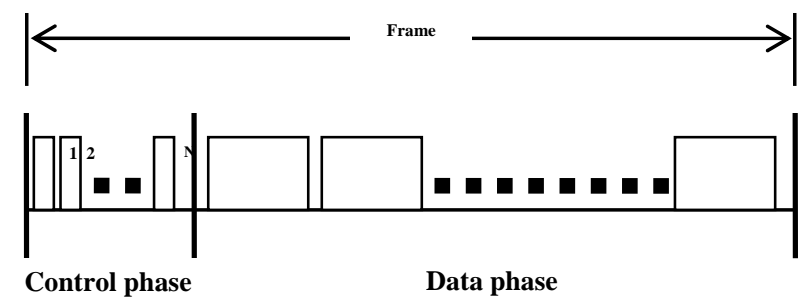

Fig.1. Frame structure.

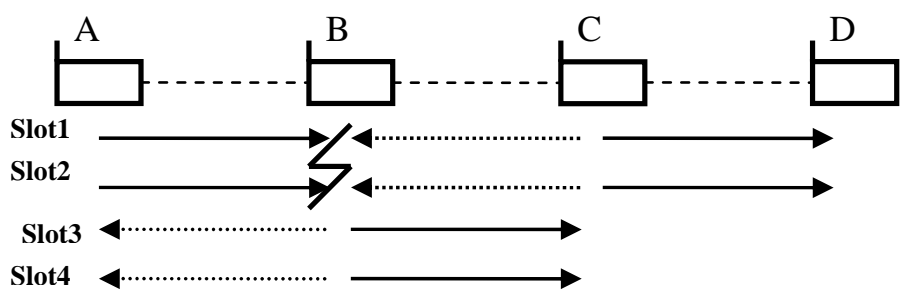

Fig. 2. Hidden terminal problem.[6]

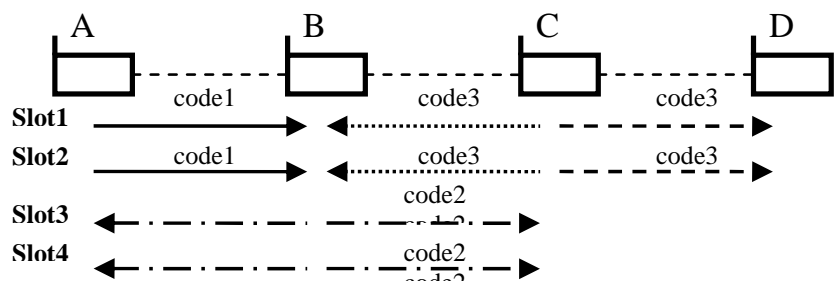

Fig. 3. CDMA over TDMA.

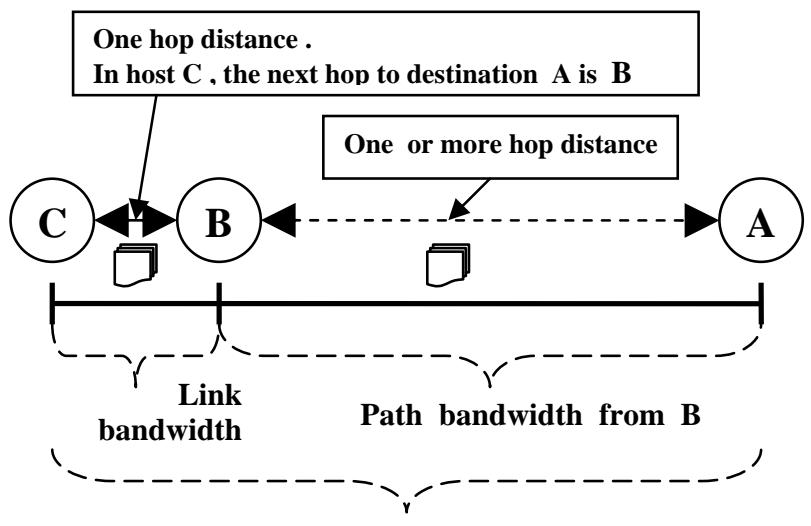

Calculate bandwidth from $\mathrm{C}$ to $\mathrm{A}$

Fig. 4. Bandwidth information calculation overview.

Table1

\begin{tabular}{|l|l|}
\hline ROUTE_REQUEST (RREQ) & Send to discover route \\
\hline ROUTE_REPLY (RREP) & Send to reserve route \\
\hline RESERVE_FAIL & Nack for unsuccessful reservation \\
\hline ROUTE_BROKEN & Nack for route broken \\
\hline CLEAN_RREQ & Clean surplus RREQs \\
\hline NO_ROUTE & Nack for finding no route \\
\hline DATA & Use to transport datagram \\
\hline
\end{tabular}




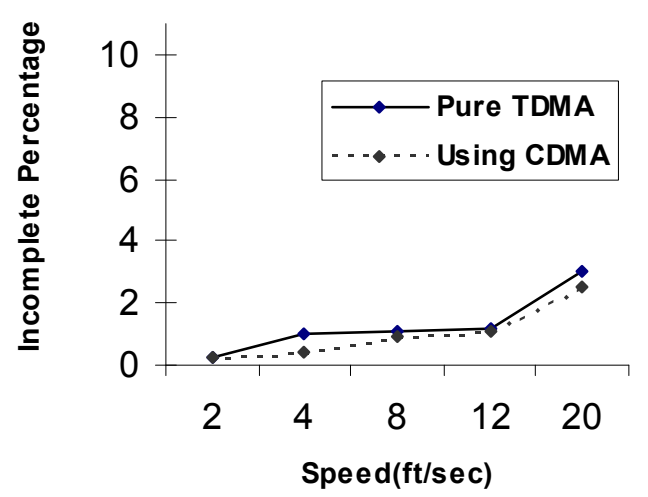

Fig. 5. Incomplete ratio of QoS1.

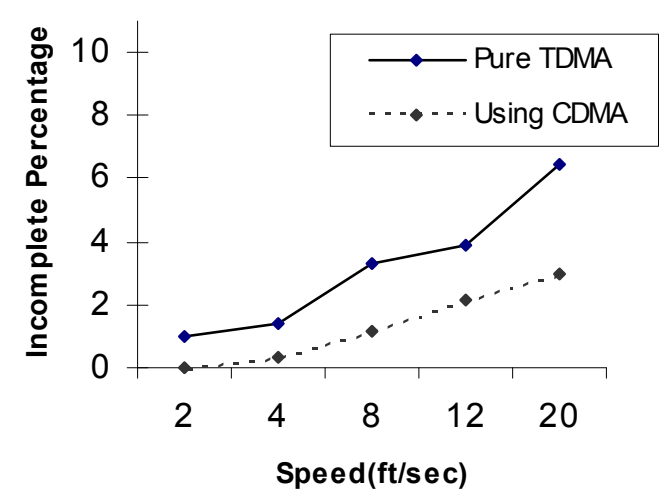

Fig. 7. Incomplete ratio of QoS1.

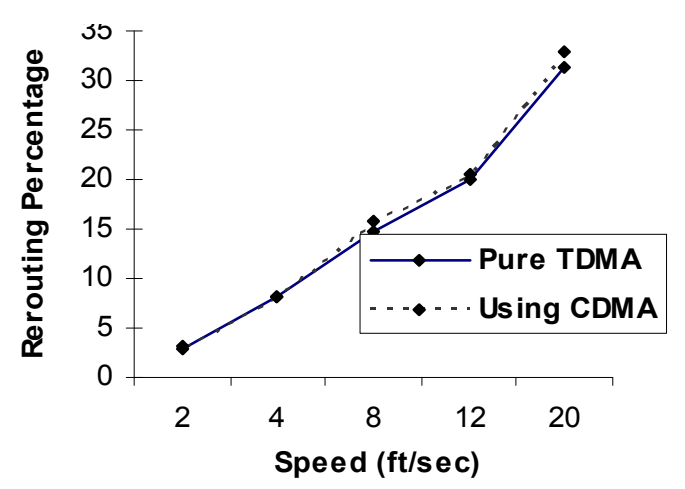

Fig. 9. Rerouting ratio of QoS 1

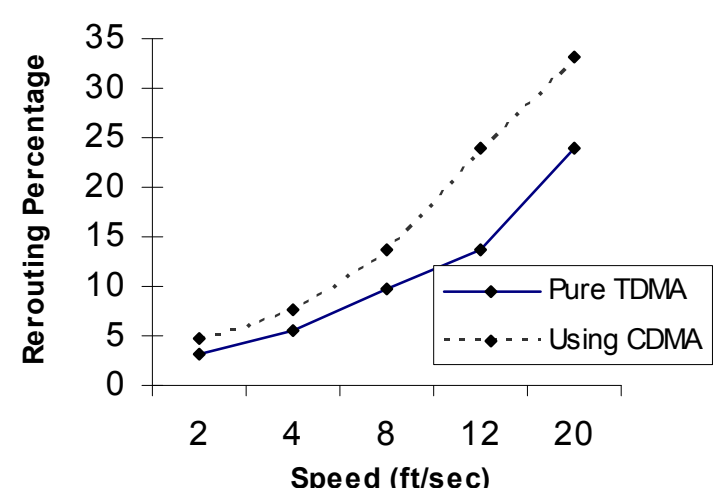

Fig. 11. Rerouting ratio of QoS4

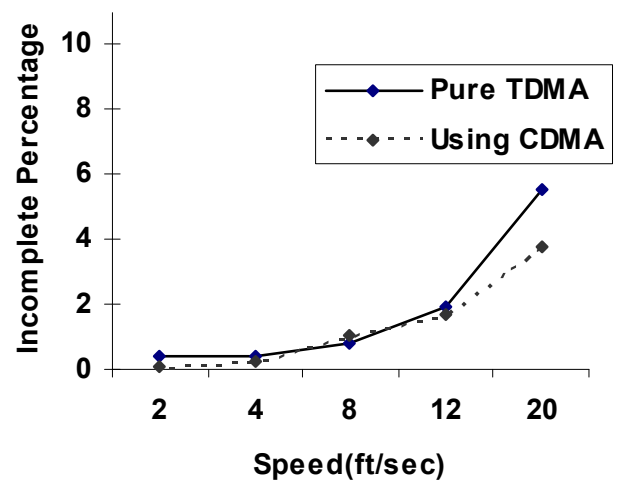

Fig. 6. Incomplete ratio of QoS2

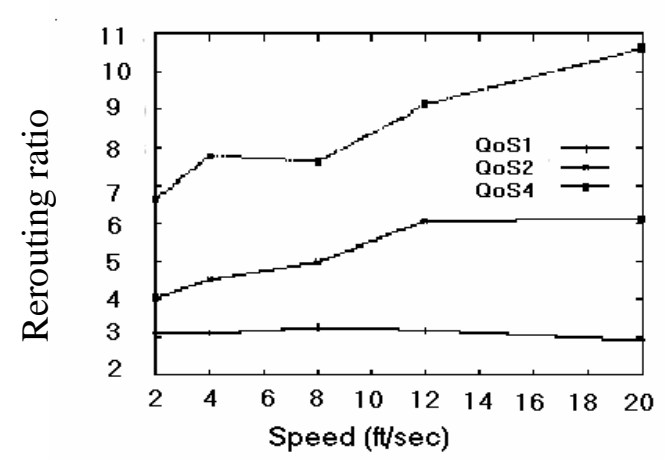

Fig. 8. Incomplete ratio of different QoS's

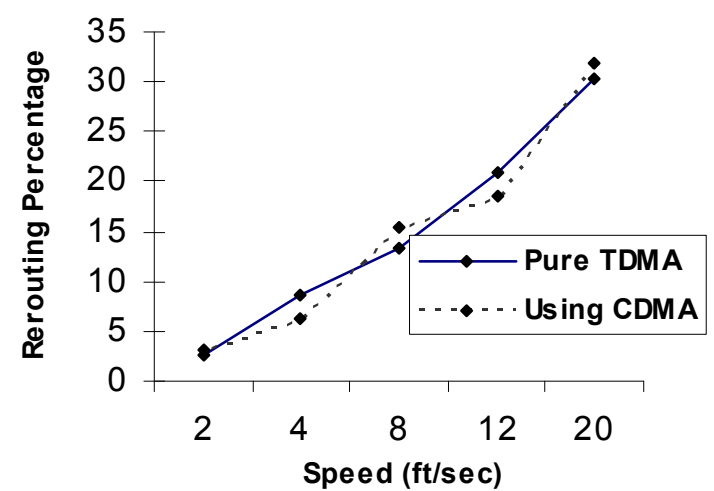

Fig.10. Rerouting ratio of QoS2.

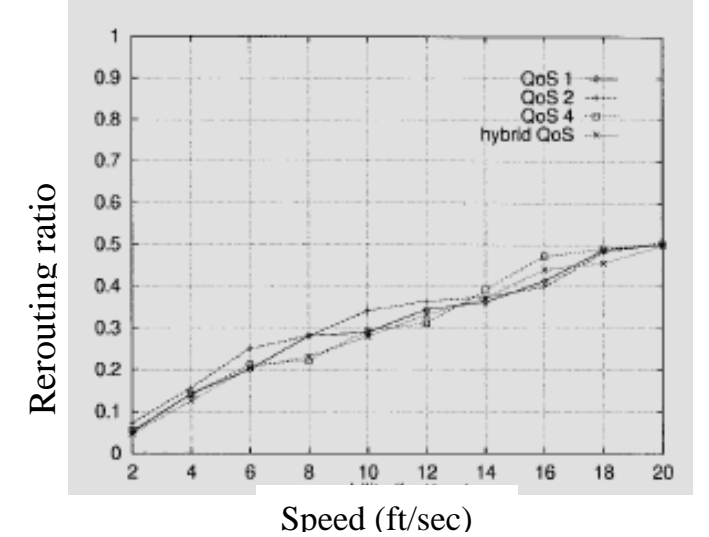

Fig.12. Rerouting ratio of different QoS's 


\section{REFRENCES}

[1] C.E. Perkins. "Ad Hoc On Demand Distance Vector (AODV) routing", InternetDraft, draft-ietf-manet-aodv-00.txt, November 1997.

[2] Chunhung Richard Lin and Jain-Shing Liu, "QoS Routing in Ad Hoc Wireless Networks", IEEE Journal on Selected Areas in Communications ,Vol. 17, No. 8, pp. 1426-1438, August 1999.

[3] Chunhung Richard LIN," On-Demand QoS Routing in Multihop Mobile Networks "Department of Computer Science and Engineering, National Sun Yat-Sen University, TAIWAN, 2001

[4] Y. Ofek, "Generating a Fault Tolerant Global Clock Using High-Speed Control Signals for the MetaNet Architecture,'IEEE Transactions on Communications, 42(5), pp. 2179-88, 1994

[5] Y.-C. Hsu and T.-C. Tsai, "Bandwidth Routing in Multihop Packet Radio Environment", Proceedings of the 3rd International Mobile Computing Workshop, March 1997.

[6] Yuh-Shyan CHEN and Yu-TingYU, " Spiral-Multi- Path QoS Routing in a Wireless Mobile Ad Hoc Network", IEICETRANS.COMMUN., VOL.E87B,NO.1JANUARY 2004

[7] David A. Maltz, "On-Demand Routing in Multi-hop Wireless Mobile Ad-hoc Networks" Ph.D. Thesis, School of Computer Science Carnegie Mellon University, May 2001. 